\title{
ON FIXED RINGS OF AUTOMORPHISMS
}

\author{
EDMUND PUCZYLOWSKI
}

\begin{abstract}
In this note we present simple proofs of some well-known results on fixed rings of finite automorphism groups of associative rings.
\end{abstract}

In this note $G$ denotes a finite group acting as automorphisms on an associative ring $R, R^{G}=\left\{x \in R \mid x^{g}=x\right.$ for all $\left.g \in G\right\}$ and $t: R \rightarrow R^{G}$ is the trace map, i.e. $t(x)=\sum_{g \in G} x^{g}$ for any $x \in R$. By $|G|$ we denote the order of $G$ and by $J$ the Jacobson radical.

In this note we present elementary proofs of the following important results.

THEOREM A (BeRgMAN AND IsAACs [1]). There exists a positive integer $n(G)$ (depending only on $G$ ) such that if $r(R)=\{x \in R \mid t(R) x=0\}$ then $(|G| r(R))^{n(G)}=$ 0 .

THEOREM B (MARTINDALE [3]). $|G| J\left(R^{G}\right) \subseteq J(R)$.

REMARK 1. (a) Bergman and Isaacs proved that

$$
n(G) \leqslant \prod_{m=1}^{|G|}\left(\left(\begin{array}{c}
|G| \\
m
\end{array}\right)+1\right) .
$$

(b) Simple induction arguments applied to Theorem A show that if $R^{1}$ is the usual extension of $R$ to a ring with unity, then for any positive integer $d$,

$$
(|G| R)^{(n(G)+1)^{d}} \subseteq|G| R^{1} t(R)^{d} R
$$

(cf. [1, Lemma 2.2]).

We need the following results.

I [2, Proposition 3.6.1]. The intersection of a finite number of modular maximal right ideals of a ring $A$ is a modular right ideal of $A$.

II [4]. If the polynomial ring $A(X, x)=(A\{\{X\}\})[x]$ in an indeterminate $x$ over the power series ring $A\{\{X\}\}$ in a set $X$ of at least two noncommutative indeterminates is $J$-radical, then the ring $A$ is nilpotent.

REMARK 2. Result II is particularly easy if card $X=$ card $A$. Indeed, if $A(X, x) \in J$ then $A\{\{X\}\}$ is nil (cf. [2]). Since card $X=\operatorname{card} A$ we can label the indeterminates in $X$ as $\left\{x_{r} \mid r \in R\right\}$. Now for some $n,\left(\sum_{r \in A} r x_{r}\right)^{n}=0$. This implies $A^{n}=0$.

LEMMA 1. If $M$ is a maximal modular right ideal of $R$ then for some $e \in R$ and all $a \in R, t(e) a-|G| a \in M$.

Received by the editors May 16, 1983.

1980 Mathematics Subject Classification. Primary 16A72, 16A22.

(O)1984 American Mathematical Society $0002-9939 / 84 \$ 1.00+\$ .25$ per page 
Proof. By I, $\bar{M}=\bigcap_{g \in G} M^{g}$ is a modular right ideal of $R$, so for some $e \in R$ and all $x \in R$, ex $-x \in \bar{M}$. Since $\bar{M}$ is $G$-invariant we conclude that $e^{g} a-a \in \bar{M}$ for $g \in G$ and $a \in R$. Consequently, $t(e) a-|G| a \in \bar{M} \subseteq M$ for $a \in R$.

Proof OF THEOREM A. If the statement does not hold then for any positive integer $n$ there exists a ring $R_{n}$ such that $\left(|G| r\left(R_{n}\right)\right)^{n} \neq 0$. Let $R=\oplus R_{n}$ be the discrete direct sum of rings $R_{n}$. Extend $G$ to act on $R$ componentwise. Then $r(R)=\bigoplus r\left(R_{n}\right)$, so $|G| r(R)$ is not nilpotent. Now extending the action of $G$ from $R$ to $R(X, x)$ in the natural way we obtain $r(R)(X, x)=r(R(X, x))$. By Lemma 1 ,

$$
|G| r(R)(X, x)=|G| r(R(X, x)) \subseteq J(R(X, x)) .
$$

Thus II implies $|G| r(R)$ is nilpotent, a contradiction.

LEMMA 2. If $t(R) \in J$ then $|G| R \in J$.

Proof. If $|G| R \notin J$ then $|G| R$ contains a maximal modular right ideal $M$. Applying Lemma 1 to $|G| R$ we obtain that for some $e \in R$ and all $a \in R$, $|G|^{2}(t(e) a-a) \in M$. Now treating $t(e) a-a$ as elements of $R^{1}$ we obtain $|G|^{2}(t(e)-1) R \subseteq M$. Since $t(R) \in J, t(e)-1$ is invertible in $R^{1}$. This and the fact that $R$ is an ideal of $R^{1}$ imply $(t(e)-1) R=R$. Consequently, $|G|^{2} R \subseteq M$. This is impossible as $M /|G|^{2} R$ is a maximal modular right ideal of $A=|G| R /|G|^{2} R$ and $A^{2}=0$.

REMARK 3. Lemma 2 applied to $R(X, x)$ and II imply that if $t(R)$ is nilpotent then so is $|G| R$ (cf. [1, Proposition 2.3]). This result is also a consequence of Remark 1(b).

Proof of Theorem B. Let $R_{1}=J\left(R^{G}\right) R^{1}$. It is clear that $R_{1}$ is a $G$-invariant right ideal of $R$ and $t\left(R_{1}\right) \subseteq J\left(R^{G}\right)$. Since $t\left(R_{1}\right)$ is an ideal of $R^{G}, t\left(R_{1}\right) \in J$. Hence by Lemma 2, $|G| R_{1} \subseteq J(R)$. Consequently, $|G| J\left(R^{G}\right) \subseteq J(R)$.

REMARK 4. Let $P$ and $L$ denote the prime and the locally nilpotent radicals, respectively. It is known [4] that $J(R(X, x)) \neq 0$ if and only if $P(R) \neq 0$ and that $J(R\{X\})=L(R)\{X\}$, where $R\{X\}$ is the polynomial ring in a set $X$ of at least two noncommutative indeterminates over $R$. This, along with II and the respective results concerning $J$ imply

(a) if $t(R) \in P(t(R) \in L)$ then $|G| R \in P(|G| R \in L)$,

(b) $|G| P\left(R^{G}\right) \subseteq P(R)$ and $|G| L\left(R^{G}\right) \subseteq L(R)$.

Consequently, if $R$ contains no $|G|$-torsion then $P\left(R^{G}\right)=P(R) \cap R^{G}$ and $L\left(R^{G}\right)$ $=L(R) \cap R^{G}$.

\section{REFERENCES}

1. G. M. Bergman and I. M. Isaacs, Rings with fixed-point-free group actions, Proc. London Math. Soc. 27 (1973), 69-87.

2. N. Jacobson, Structure of rings, Amer. Math. Soc. Colloq. Publ., vol. 37, Amer. Math. Soc., Providence, R. I., 1964.

3. W. S. Martindale, Fixed rings of automorphisms and the Jacobson radical, J. London Math. Soc. 17 (1978), 42-46.

4. E. R. Puczylowski, Radicals of polynomial rings, power series rings and tensor products, Comm. Algebra 8 (1980), 1699-1709.

Institute of Mathematics, University of WarsaW, Warsaw, Poland 